\title{
Diacronie
}

Studi di Storia Contemporanea

$\mathrm{N}^{\circ} 18,2 \mid 2014$

Le esposizioni: propaganda e costruzione identitaria

\section{«La Civiltà Cattolica» e le esposizioni torinesi (1884 e 1898)}

\section{Carlo Bovolo}

\section{(C) OpenEdition}

\section{Journals}

\section{Edizione digitale}

URL: http://journals.openedition.org/diacronie/1493

DOI: 10.4000/diacronie. 1493

ISSN: 2038-0925

\section{Editore}

Association culturelle Diacronie

\section{Notizia bibliografica digitale}

Carlo Bovolo, « «La Civiltà Cattolica» e le esposizioni torinesi (1884 e 1898) », Diacronie [Online], № 18,

2 | 2014, documento 12, Messo online il 01 juin 2014, consultato il 19 avril 2019. URL : http://

journals.openedition.org/diacronie/1493; DOI : 10.4000/diacronie.1493 


\section{Diacronie}

12/

\section{«La Civiltà Cattolica» e le esposizioni torinesi (1884 e 1898)}

Carlo BOVOLO*

L'articolo analizza lo sguardo della «Civiltà Cattolica», rivista dei gesuiti, sulle Esposizioni generali italiane svoltesi a Torino nel 1884 e nel 1898. In entrambe le esposizioni un ruolo centrale fu attribuito ai processi di costruzione della nazione $e$ delle identità nazionali, all'organizzazione del consenso verso le élite dirigenti e la monarchia, attraverso un uso politico della memoria risorgimentale, oltre alla celebrazione del progresso. Nel 1884 la rivista respinse i valori laici e liberali di un'esposizione non solo tecnico-scientifica ma anche politica, rivendicando invece il contributo e la legittimità dei cattolici nelle scienze; nel 1898 la polemica, seppur presente, fu attenuata dall'affiancamento di iniziative cattoliche a quelle laiche $e$ liberali.

\section{1. «La Civiltà Cattolica», strumento al servizio dell'apologia}

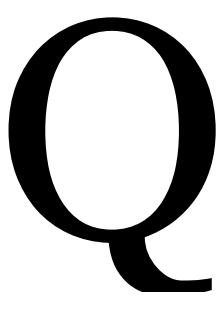
uando nel 1851 il Crystal Palace di Londra ospitò la prima Esposizione universale, «La Civiltà Cattolica», rivista dei gesuiti italiani, era al secondo anno di vita, essendo stata fondata l'anno precedente da alcune figure di primo piano dell'ordine di Gesù, come Carlo Maria Curci, Luigi Taparelli d'Azeglio, Matteo Liberatore, Antonio Bresciani, e con un convinto consenso del pontefice Pio IX. 
«Specchio fedele degli indirizzi della Santa Sede»1 e «laboratorio privilegiato del pensiero cattolico intransigente $»^{2}$, «La Civiltà Cattolica» nacque come un periodico in lingua italiana, a cadenza quindicinale, di alta divulgazione e ampia diffusione, «che combattesse gli errori moderni e, allo stesso tempo, difendesse dagli attacchi dei liberali e dei razionalisti la dottrina cattolica e gli interessi della Santa Sede»3. La scelta della rivista quale più valido e funzionale mezzo di diffusione e difesa dei principi cattolici e strumento di formazione di un pubblico cattolico colto e aggiornato, si dovette alla consapevolezza che «il così detto giornalismo è di fatto in questi giorni il ramo di stampa più fecondo di scrittura, e il più efficace alla diffusione ed alla tutela dei sacri principi»4, come riconobbero gli stessi redattori nel primo numero. A partire dal 1850 la rivista dei gesuiti condusse una battaglia in difesa dei principi della Rivelazione e dei dogmi della Chiesa, in tutti gli ambiti, politico, religioso, sociale, culturale e scientifico. «La Civiltà Cattolica» giocò un ruolo di primo piano nel panorama italiano dei periodici della seconda metà dell'Ottocento, divenendo un punto di riferimento per l'opinione pubblica cattolica di tutta la penisola (in pochi anni, infatti, raggiunse oltre 10.00o abbonamenti e una diffusione pressoché "nazionale"). Strumento di apologia e propaganda tra $\mathrm{i}$ più significativi in mano alla Chiesa 5 , sulle sue pagine furono commentati tutti gli eventi degni di nota dell'Italia in formazione prima e di quella unita poi, vennero pubblicati soprattutto approfondimenti teologici, polemiche politiche, racconti didascalici, articoli di grande spessore culturale, ma anche rubriche di attualità, scienze e archeologia, elenchi di letture consigliate, provvedimenti della Chiesa.

Tra i diversi argomenti trattati, i temi riguardanti scienze e tecnologia ottennero un spazio significativo sulle pagine della rivista. L'interesse verso tematiche scientifiche, sia per confutazioni (come nel caso dell'evoluzionismo) che per elogi (ad esempio, la

\footnotetext{
${ }^{1}$ FORNO, Mauro, Informazione e potere: storia del giornalismo italiano, Roma-Bari, Laterza, 2012, p. 20.

2 TRANIELLO, Francesco, Religione cattolica e Stato nazionale: dal Risorgimento al secondo dopoguerra, Bologna, Il Mulino, 2007, p. 96.

3 SALE, Giovanni, «La Civiltà Cattolica» nella crisi modernista (1900-1907), Milano, Jaca Book, 2001, p. 37. Sulla "Civiltà Cattolica» cfr.: DANTE, Francesco, Storia della "Civiltà Cattolica» (1850-1891): il laboratorio del papa, Roma, Studium, 1990; DE ROSA, Giuseppe, Introduzione, in ID. (a cura di), La Civiltà Cattolica 1850-1945, Roma, Landi, 1971, pp. 9-101; ID., La Civiltà Cattolica: 150 anni al servizio della Chiesa 1850-1999, Roma, La Civiltà Cattolica, 1999; MUCCI, Giandomenico, Carlo Maria Curci: il fondatore della "Civiltà Cattolica», Roma, Studium, 1988; SANI, Roberto, Un laboratorio politico e culturale: «La Civiltà Cattolica», in RICCARDI, Andrea (a cura di), Pio XII, Roma-Bari, Laterza, 1984, pp. 409-436.

4 «La Civiltà Cattolica», anno I, serie I, vol. I, 1850, p. 101.

5 Il legame profondo e privilegiato della "Civiltà Cattolica» con il papato è ulteriormente sottolineato dal fatto che le bozze della rivista, prima di essere pubblicate, dovevano ottenere il beneplacito papale.
} 
conciliazione di fede e scienza), unitamente all'attenzione verso l'attualità portò i redattori della rivista a interessarsi alle esposizioni, fin dal quella londinese del 1851. Simboli del positivismo, che gradualmente si stava diffondendo anche in Italia, oggetto di discussione e interesse della stampa italiana e internazionale e di curiosità da parte dell'opinione pubblica, le esposizioni si prestavano relativamente bene alla strategia, portata avanti dalla «Civiltà Cattolica» fin dai primi numeri, una strategia di uso apologetico e apostolico della tematiche scientifiche in senso lato, i cui capisaldi erano la difesa della Rivelazione dagli errori del secolo, la diffusione di tesi che conciliassero scienza e fede, tema ritenuto di primaria importanza, la volontà di tenersi aggiornata sulle novità scientifiche e tecnologiche e la conseguente volontà di controllo sulle diverse discipline e la rivendicazione di un ruolo significativo e legittimo dei cattolici (anche e soprattutto ecclesiastici) in ogni questione e dibattito scientifico ${ }^{6}$.

Come già accennato sopra, la rivista si interessò in più di un'occasione alle esposizioni, a partire dalla prima Esposizione universale di Londra, su cui informò i lettori dei preparativi e dell'inaugurazione nella rubrica dedicata ai fatti di cronaca. Se l'interesse della «Civiltà Cattolica» si limitò per certi aspetti a uno stringato dovere cronachistico verso le esposizioni in programma al di là delle Alpi, per quanto riguarda quelle italiane l'attenzione fu sicuramente maggiore. Nello specifico, la rivista si focalizzò sulle due esposizioni torinesi del 1884 e del 1898, due eventi considerati di particolare rilevanza per diversi motivi.

In primo luogo, la sede di queste due esposizioni aveva una portata simbolica molto forte: Torino, culla del positivismo italiano e prima capitale di un Regno d'Italia nato in aperto contrasto con la Chiesa. La città vantava, inoltre, una decennale tradizione di esposizioni dell'arte e dei prodotti dell'industria, eventi spesso non disgiunti da finalità miranti a suscitare consenso dinastico e costituire un'identità subalpina7 . La tradizione

6 BOVOLO, Carlo, Scienza e scienziati nella «Civiltà Cattolica»1850-191o, Tesi di laurea in Storia del Risorgimento, Università degli Studi di Torino, Torino, a.a. 2012/2013.

7 Tra i più recenti studi sulle esposizioni torinesi si possono citare: BASSIGNANA, Pier Luigi (a cura di), Le esposizioni torinesi: nei documenti dell'Archivio Storico Amma 1829-1898, Torino, Umberto Allemandi, 1992; ID., Torino effimera: due secoli di grandi eventi, Torino, Umberto Allemandi, 2006; COMOLI MANDRACCI, Vera, Torino città di loisirs: viali, parchi e giardini tra Otto e Novecento, Torino, Archivio Storico Città di Torino, 1996; LEVRA, Umberto, ROCCIA, Rosanna (a cura di), Le esposizioni torinesi 1805-1911: specchio del progresso e macchina del consenso, Torino, Archivio Storico Città di Torino, 2003; MONTALDO, Silvano, Le esposizioni industriali nel Regno di Sardegna: suggestioni modernizzanti tra propaganda dinastica e riforme economiche, in BIGATTI, G, ONGER, S (a cura di), Arti, tecnologia, progetto. Le esposizioni d'industria in Italia prima dell'Unità, Milano, Franco Angeli, 2007, pp. 107-150; ABBATTISTA, Guido, Umanità in mostra: esposizioni etniche e invenzioni esotiche in Italia (1880-1940), Trieste, Edizioni Università di Trieste, 2013. Una più completa e puntuale bibliografia sull'argomento, approntata dalle Biblioteche Civiche Torinesi e comprendente anche le pubblicazioni coeve, è consultabile online, URL: 
delle esposizioni torinesi ebbe origine durante il periodo napoleonico, quando nell'aprile 1805 Torino, capoluogo di un territorio annesso all'impero francese, ospitò un evento organizzato sul modello dell'esposizione parigina del 1798 . Il risultato fu un'esposizione di carattere misto (tratto ricorrente nelle esposizioni sabaude), dove accanto ai modesti prodotti delle manifatture piemontesi, operanti in un contesto ancora preindustriale e fortemente limitato dalla subalternità economica francese, trovò spazio una sezione di belle arti, celebranti l'imperatore e il regime da lui impersonato ${ }^{8}$. Durante la Restaurazione, non mancarono altre esposizioni: se, da una parte, sotto il regno di Carlo Felice vennero privilegiate le belle arti e una visione ancora corporativa delle arti e dei mestieri, dall'altra, nell'età carloalbertina, si riprese dal precedente napoleonico un uso delle esposizioni funzionale alla celebrazione della dinastia e al rafforzamento dell'istituto monarchico e del suo legame con il popolo ${ }^{9}$. Fu però dall'esposizione del 1844 (e nelle successive del 1850 e del 1858) che il progresso industriale giocò un ruolo di primo piano: pur non venendo mai meno, l'apologia dinastica e la rassegna celebrativa si mutarono in una sorta di «nazionalismo economico ${ }^{10}$, incentrato sullo sviluppo industriale e produttivo e sulla conoscenza e diffusione delle innovazioni. A causa del trasferimento della capitale da Torino a Firenze nel 1864 e della conseguente crisi, la città conobbe un ritorno delle esposizioni artistiche e ospitò alcuni modesti tentativi di esposizioni industriali e settoriali. Fu con le due manifestazioni del 1884 e del 1898 che Torino riacquistò la centralità, quale sede ideale per la duplice celebrazione del Risorgimento nazionale e del progresso dell'industria e della tecnica.

In secondo luogo, entrambe le manifestazioni posero il tema della nazione al centro delle celebrazioni: nel 1884 fu l'occasione per celebrare per la prima volta una visione di un Risorgimento «nazional-popolare» ${ }^{11}$, onnicomprensivo e conciliatorista, secondo la linea politica di Francesco Crispi e Tommaso Villa; quattordici anni dopo la ricorrenza del cinquantesimo anniversario dello Statuto Albertino celebrò l'epopea nazionale e i primi decenni di vita dello Stato unitario. Entrambe le manifestazione erano dunque finalizzate a creare e rafforzare un'identità nazionale, a suscitare

http://www.comune.torino.it/cultura/biblioteche/ricerche_cataloghi/pdf/bibliografie/esposizi oni.pdf $>$ [consultato il 2 aprile 2014].

8 CAVICCHIOLI, Silvia, Tra Sette e Ottocento, in LEVRA, Umberto, ROCCIA, Rosanna (a cura di), Le esposizioni torinesi 1805-1911, cit., pp. 3-31.

9 BASSIGNANA, Pier Luigi, Il messaggio dell'età carloalbertina, in LEVRA, Umberto, ROCCIA, Rosanna (a cura di), Le esposizioni torinesi 1805-1911, cit., pp. 33-51.

10 Ibidem.

${ }^{11}$ Cfr. LEVRA, Umberto, Fare gli italiani: memoria e celebrazione del Risorgimento, Torino, Comitato di Torino dell'Istituto per la Storia del Risorgimento Italiano, 1992. 
consenso verso le classi dirigenti nazionali e cittadine così come verso la monarchia e a favorire la pacificazione sociale.

Infine, l'attenzione rivolta dalla "Civiltà Cattolica» alle due esposizioni subalpine risulta particolarmente interessante anche perché il periodico dei gesuiti diede degli eventi uno sguardo e una lettura esterni rispetto all'ambiente torinese, pur condividendo le posizioni critiche di periodici cattolici torinesi, primo tra tutti «L'Italia Reale» ${ }^{12}$.

\title{
2. «Non tutti si curano o amano di prendere parte a siffatte mostre»: l'Esposizione Generale Italiana del 1884
}

Il contesto in cui si svolse l'Esposizione Generale Italiana del 1884 era radicalmente mutato rispetto a quello delle precedenti esposizioni torinesi: fatta l'Italia, si era nel pieno del processo etichettato con il d'azegliano «fare gli italiani», vale a dire la creazione nel neonato Stato unitario di un'identità nazionale condivisa e omogenea che avesse le proprie radici e la propria legittimazione nel Risorgimento. Sempre più influenzata dalle grandi esposizioni internazionali, l'esposizione del 1884 fece emergere anche a Torino quegli elementi che già circolavano nella cultura europea a proposito di tali iniziative, come evidenziato da Umberto Levra e Rosanna Roccia:

\begin{abstract}
Erano il triplice obiettivo di organizzazione del consenso alla nazione, di inventario delle forze produttive e di adesione alla politica dei ceti di governo; la finalità didascalica e didattica, per plasmare ai valori borghesi quelle classi lavoratrici che premevano dal basso per partecipare alla decisioni relative alla cosa pubblica; l'ideologia interclassista, alla ricerca illusoria della pace sociale e di un equilibrio perfetto tra capitale e lavoro; la celebrazione della borghesia attraverso l'esaltazione del progresso e la spettacolarità dei prodigi della scienza e della tecnica ${ }^{13}$.
\end{abstract}

L'Esposizione Generale Italiana del 1884 ebbe un grande successo, sfiorando i 3 milioni di visitatori, e fu caratterizzata da un evidente e insistente nesso tra i padiglioni dell'industria e della tecnica e la sezione dedicata al Risorgimento, «onde collegare senza soluzioni di continuità il mito laico della scienza e del progresso con il presupposto storico e nazionale di esso, cioè il patrimonio di valori dell'epopea unitaria

12 Il tema del rapporto tra «La Civiltà Cattolica» e «L'Italia Reale», quotidiano torinese di primo piano tra i periodici cattolici, risulta particolarmente interessante e meritevole di ulteriori approfondimenti e ricerche.

13 LEVRA, Umberto, ROCCIA, Rosanna (a cura di), Le esposizioni torinesi 1805-1911, cit., p. XVI. 
posto alla base dell'identità stessa della patria comune» ${ }^{14}$, secondo la linea dettata da Tommaso Villa, animatore dell'iniziativa e presidente del Comitato esecutivo ${ }^{15}$.

All'esposizione del 1884, che univa dunque celebrazione del progresso e del Risorgimento, fu ovviamente assente la Chiesa in forma ufficiale, anche se non mancarono singoli esponenti del clero o di ordini e congregazioni ${ }^{16}$. Nelle specifico si distinsero i Salesiani, tradizionalmente forti in Piemonte grazie all'azione di Don Bosco, che presentarono in un padiglione il ciclo della fabbricazione della carta, con l'ausilio di moderni macchinari: chiusa la domenica, però, l'esposizione salesiana rappresentava idealmente un universo a sé, in cui l'enfasi era posta sul mestiere e sull'apprendimento tecnico e con una posizione critica sul progresso e sulla nascente società industriale ${ }^{17}$.

«La Civiltà Cattolica» informò i lettori dell'inaugurazione dell'esposizione nella sezione dedicata all'Italia della rubrica di attualità Cronaca contemporanea pubblicata nel fascicolo del 10 maggio 1884, senza rinunciare alla polemica:

L’inaugurazione non riuscì né splendida, né imponente, come si desiderava, perché la pioggia, che veniva giù a secchie, convertì in una vera pozza il vasto piazzale che è davanti all'ingresso e tolse alla festa del lavoro, come la chiamano i suoi panegiristi, quel gaio aspetto che gioconda lo sguardo degli spettatori. D'altra parte i lavori erano abbastanza indietro ${ }^{18}$.

Il critico resoconto dell'inaugurazione, che «per ragion della fretta non ebbe nulla di grandioso, e nulla ancora di bello»19, proseguiva sdegnandosi di fronte ai discorsi pronunciati per l'occasione, al cospetto di Umberto I, dal principe Amedeo di Savoia, da Villa e da Bernardino Grimaldi, ministro dell'Agricoltura, Industria e Commercio, dal momento che «nessuno parlò di Dio!»²0. Che non vi facessero accenno due liberali come Villa e Grimaldi non stupiva affatto la rivista, ma «che si fosse astenuto

${ }^{14}$ LEVRA, Umberto, Fare gli italiani, cit., p. 152.

15 Tommaso Villa (1832-1915), avvocato penalista di successo e massone, vicino politicamente a Francesco Crispi, fu per decenni impegnato in una fitta attività politica, sia a Torino come consigliere comunale, sia a Roma come deputato e poi senatore e come ministro dell'Interno (1879) e ministro della Giustizia (1879-1881) nei governi Cairoli e Presidente della Camera dei Deputati dal 1895 al 1895 e poi di nuovo dal 1900 al 1902. Cfr. MONTALDO, Silvano, Patria e affari: Tommaso Villa e la costruzione del consenso tra Unità e Grande Guerra, Torino, Comitato di Torino dell'Istituto per la Storia del Risorgimento Italiano, 1999.

16 BIGINELLI, Luigi, Il clero all'Esposizione Nazionale di Torino. Reminiscenze del Sac. Biginelli Teolog. Luigi, Direttore dell'Ateneo religioso, periodico illustrato, Napoli, Tipografia degli Accattoncelli, 1884.

17 AIMONE, Linda, FILIPPI, Francesca, 1884. La nazione italiana al lavoro, in LEVRA, Umberto, ROCCIA, Rosanna (a cura di), Le esposizioni torinesi 1805-1911, cit., p. 94.

18 «La Civiltà Cattolica», anno XXXV, serie XII, vol. VI, 1884, p. 494.

19 «La Civiltà Cattolica», anno XXXV, serie XII, vol. VI, 1884, p. 495.

${ }^{20}$ Ibidem. 
d'invocarlo un Principe di Casa Savoia, questo è stato per tutti argomento di altissimo stupore»21. Paradossalmente, fu il diplomatico tedesco Keudell, «rappresentante di un Governo luterano», a nominare la Provvidenza e ad affermare l'importanza della religione nella storia d'Italia. Mentre all'esposizione di Milano del 1881 si era evitato di toccare tematiche politiche, «a Torino invece non si è avuto in vista che la politica, la quale si è fatta entrare sin nei più minuti accessori della Esposizione; e ciò ha nociuto all'effetto che si volea produrre»22. L'articolo si concludeva con un giudizio inappellabile, che non solo rifiutava gli ideali politici e culturali di cui l'esposizione si faceva portatrice, ma anche sollevava dubbi sull'opportunità economica della manifestazione: «è stato male malissimo l'aver cacciato dentro i padiglioni della mostra la politica; perché l'Italia in quella mostra giuoca una gran carta e Torino il suo credito. Azionisti e espositori giuocano danari e riputazione, tutti corrono un grande rischio» ${ }^{23}$.

Bocciata l'esposizione per il connubio tra progresso nell'industria e nella tecnica e celebrazione del Risorgimento, la rivista volse dunque la propria attenzione sulla parte scientifica dell'evento. Nella rubrica Scienze naturali, curata dal gesuita Francesco Salis Seewis e pubblicata l'8 novembre 1884, si rivendicò il rilevante contributo scientifico e tecnologico del clero: «la nostra Appendice va tutta ad onore dei preti. Ma che colpa ne abbiamo noi se cotesto Clero ignorante e trascurato finisce poi sempre con estorcere elogi e premii ancora da malevoli dovunque è qualche mostra o concorso scientifico? Un esempio recentissimo ce ne ha dato l'Esposizione ultima di Torino» ${ }^{24}$. Seguiva quindi un lungo elenco di ecclesiastici distintisi nelle diverse discipline:

Nelle gallerie di fisica terrestre, astronomia, meteorologia, gli scienziati ecclesiastici riportarono la palma senza meno. Parecchi di loro come il P. Cecchi scolopio, il P. Denza barbabita, il prof. Tono direttore dell'Osservatorio del Seminario Patriarcale di Venezia riportarono medaglie d'oro. Si fecero ammirare il P. Serpieri, il Faa di Bruno, il Mercalli, il Varsico, il Fulcis, il Roccis, il Quandel, il Medichini, il Carrel, il Capanni, il Bianchi, il Maccalini, il Groglio, il Flecchia. Al lettore non sarà sfuggito che mancano qui non pochi nomi di ecclesiastici ben conosciuti nel mondo scientifico: ché non tutti si curano o amano prendere parte a siffatte mostre ${ }^{25}$.

In questo modo la rivista, prese le distanze e rigettato il messaggio politico dell'esposizione, si dedicò alla parte scientifica, all'apparenza politicamente neutra: ciò

\footnotetext{
${ }^{21}$ Ibidem.

22 Ibidem.

23 Ibidem.

24 «La Civiltà Cattolica», anno XXXV, serie XII, vol. VIII, 1884, pp. 489-490.

25 Ibidem.
} 
le consentì di rivendicare l'importanza e la legittimità del contributo scientifico e tecnico dei cattolici e, allo stesso tempo, di presentare ai lettori l'auspicabile e doverosa conciliazione di scienza e fede, un tema spesso ricorrente sulle pagine del periodico:

Qual è la classe di dotti, medici, avvocati, giureconsulti, militari, ingegneri, matematici, ecc., che possa vantare un egual numero di uomini segnalati in qualche scienza estranea a quella della loro particolar professione, quanti ne porge la classe degli ecclesiastici? V'ha egli tanti avvocati naturalisti quanti v'ha preti naturalisti? V'ha tanti medici che allo studio e alla conoscenza della medicina abbiano accoppiato e aggiunto quello dell'astronomia, quanti sono i preti che coompiuto lo studio lungo e scabroso della teologia vi hanno aggiunto quello della scienza degli astri, fino a segnalarsi fra gli astronomi? E così si dica del rimanente. Aspettiamo la risposta da coloro che accusano o ripiangono l'ignoranza del Clero $^{26}$.

Terminata l'esposizione, «La Civiltà Cattolica» tornò a polemizzare aspramente sull'organizzazione dell'evento e sui suoi contenuti politici e culturali nell'articolo intitolato Il come e il perché della mostra di Torino ${ }^{27}$. Secondo i gesuiti, la mostra era stata apertamente anticattolica e antipapale, «imbevuta di massonismo» ${ }^{28}$. Come evidenzia Guido Abbattista, l'esposizione aveva dato dell'Italia un'immagine negativa a causa di «coloro stessi i quali pretesero creare un'Italia nuova, degenere da tutte le avite grandezze che furono principalmente grandezze religiose; ed ora anche una Mostra d'arti e d'industrie vollero torcere in apoteosi di quel loro gran fatto?»29. La conclusione del periodico era dunque che «tutto sommato dimostra una volta di più che questa Italia, così come coloro che l'hanno voluta, precipita ogni dì più in basso, non rimanendole di tutte le antiche sue glorie che il rammarico di averle perdute»30.

\section{3. «Feste e fame in Italia»: l'Esposizione Generale Italiana del 1898}

Come per l'edizione del 1884, anche l'Esposizione Generale Italiana del 1898 si svolse in un clima mutato rispetto alla precedente, sia a livello nazionale che a livello locale. L'occasione dell'evento era il cinquantesimo anniversario dello Statuto Albertino e del Quarantotto, per cui apparvero prioritari gli aspetti relativi alla creazione di un'identità nazionale, all'organizzazione del consenso verso la classe dirigente e la

\footnotetext{
26 Ibidem.

27 «La Civiltà Cattolica», anno XXXV, serie XII, vol. VIII, 1884, pp. 521-536.

28 «La Civiltà Cattolica», anno XXXV, serie XII, vol. VIII, 1884, p. 525.

29 ABBATTISTA, Guido, Umanità in mostra, cit., p. 212.

30 «La Civiltà Cattolica», anno XXXV, serie XII, vol. VIII, 1884, p. 536.
} 
monarchia e alla pacificazione sociale, anche e soprattutto alla luce della crisi economica e politica che stava vivendo il Paese. Proprio nel corso del 1898 il malcontento popolare raggiunse il culmine nel maggio con i moti di Milano, repressi nel sangue dal generale Bava Beccaris.

Anche a Torino la situazione era cambiata rispetto al 1884, quando Villa aveva potuto giocare un ruolo di assoluta preminenza e il successo dell'iniziativa aveva rafforzato la classe dirigente laica e liberale di cui faceva parte. Nelle elezioni amministrative del $\mathbf{1 8 9 5}$, infatti, in seguito a una campagna elettorale tesa, i cattolici torinesi ottennero un grande successo, nonostante la formazione di una giunta a maggioranza liberale, sotto la guida di Felice Rignon.

$\mathrm{Fu}$ in questo contesto che Villa lanciò la proposta di celebrare il cinquantenario dello Statuto con una grande esposizione, per dimostrare i progressi compiuti in mezzo secolo grazie alla libertà e alla scienza. Immediata fu la reazione di Stefano Scala, direttore del quotidiano «L'Italia Reale», organo dell'intransigentismo cattolico subalpino, il quale rivendicò il ruolo fondamentale della religione al fianco di libertà e scienza. La tensione politica tra cattolici e liberali si acuì, fino alle dimissioni del sindaco Felice Rignon e al commissariamento del Comune nel novembre del 1896. I cattolici torinesi, in base al cosiddetto principio di riparazione, secondo cui veniva contrapposta a ogni iniziativa anticlericale una cattolica, iniziarono dunque a organizzare un'esposizione alternativa a quella di Villa ${ }^{31}$. L'acuirsi delle tensioni sociali nel Paese facilitò una parziale riconciliazione tra le due parti, sancita da una convenzione firmata il 18 maggio 1896 da Villa e dal barone Antonio Manno, figura di spicco del élite clericale torinese e presidente del Comitato esecutivo dell'Esposizione di Arte sacra: la "paura dei rossi", condivisa da liberali e cattolici, determinò «l'inserimento dell'Esposizione di Arte sacra, delle Missioni cattoliche e delle Opere di Carità Cristiana nel tessuto stesso dell'Esposizione generale italiana. Fu un fatto del tutto nuovo, che rese la manifestazione un unicum nel panorama italiano ottocentesco e costituì un precedente nei rapporti tra politica e religione» ${ }^{2}$. La gestione degli eventi fu quindi unitaria e i 3 milioni e mezzo di visitatori complessivi furono un successo. Tuttavia, l'esposizione del 1898 «non realizzò una vera e propria ricomposizione tra l'identità cattolica e l'identità liberale laica: fu piuttosto una giustapposizione delle due anime, per certi versi sconcertante. La mostra italiana e quella cattolica vennero infatti

${ }^{31}$ MONTALDO, Silvano, Patria e religione nel 1898, in LEVRA, Umberto, ROCCIA, Rosanna (a cura di), Le esposizioni torinesi 1805-1911, cit., pp. 111-144.

$3^{2}$ MONTALDO, Silvano, Patria e religione nel 1898, cit., p. 123. 
tenute separate, ma furono al tempo stesso unite»33. Da una parte i padiglioni espositivi celebrarono i progressi compiuti in cinquant'anni di Statuto, grazie a libertà e scienza, con l'esibizione di elettricità, automobili, cinema, telegrafo, basi del futuro decollo industriale di Torino e dell'Italia. Dall'altra, l'iniziativa cattolica si costituì in più parti: l'ostensione della Sindone, con il coinvolgimento di Casa Savoia e che da sola richiamò a Torino circa 800.000 visitatori; un'Esposizione di Arte sacra; una minuziosa rassegna delle Opere di Carità Cristiana; un'esposizione etnica sulle missioni cattoliche nel mondo, a cui si aggiunsero come appendici l'inaugurazione del monumento a Don Bosco a Castelnuovo d'Asti e il terzo Congresso Mariano.

La notizia dell'inaugurazione dell'Esposizione Generale Italiana del 1898 fu data dalla «Civiltà Cattolica», nella consueta rubrica di attualità, anticipata da un breve articolo intitolato Feste e fame in Italia, una critica contrapposizione tra la celebrazione del cinquantenario dello Statuto e i tumulti che negli stessi giorni attraversavano la penisola, originati «dal fiscalismo esorbitante e dal lusso spendereccio de' nostri governanti»34. Questa contrapposizione tra la celebrazione di mezzo secolo di libertà e progresso e un'Italia reale fatta di miseria e malcontento divenne un motivo ricorrente di polemica verso l'esposizione laica e liberale: «Mentre tali fatti [i tumulti popolari] accadevano in Italia, s'apriva a Torino, il $1^{\circ}$ maggio, la grande esposizione nazionale [...]. Quanto ai discorsi, poi, tutti furono magniloquenti e sonanti, e tali che facevano un brutto contrasto con quel che stava accadendo in quell'ora stessa nel resto d'Italia»35.

Passando a parlare dell'inaugurazione dell'Esposizione di Arte sacra il registro dell'articolo mutava completamente: il redattore annunciò la benedizione data dall'arcivescovo di Torino Agostino Richelmy, alla presenza del re e della famiglia reale, a una mostra che «dicono anche i giornali liberali, è più attraente della mostra profana, e i Sovrani e i Principi se ne deliziarono»36. Per ulteriori approfondimenti e più precisi particolari, «La Civiltà Cattolica» consigliava la lettura del «lodato foglio torinese»37, «L'Italia Reale».

Il periodico dei gesuiti tornò nuovamente a parlare dell'esposizione a Torino in riferimento l'evento clou della manifestazione cattolica: «Da questo tenebrore che involse tutta l'Italia, prima colle sommosse, poi collo scioglimento delle società cattoliche, volgiamoci ora a Torino; ove per parecchi giorni brillò tanto splendore di

\footnotetext{
33 Ibidem.

34 «La Civiltà Cattolica», anno XLIX, serie XVII, vol. II, 1898, p. 482.

35 «La Civiltà Cattolica», anno XLIX, serie XVII, vol. II, 1898, p. 487.

${ }^{36}$ Ibidem.

37 Ibidem.
} 
viva e benefica luce per la solenne esposizione della Sacra Sindone»38. Dopo la dettagliata descrizione della cerimonia di ostensione, la rivista indicò la reliquia quasi come un simbolo identitario ben più forte e più radicato rispetto a quelli sventolati dai liberali, contenente «tutta la storia, la gloria e la fede dell'antico popolo e dell'antico Stato»39.

Se nel 1884 «La Civiltà Cattolica» spostò l'attenzione dalla celebrazione di valori laici del Risorgimento e del progresso all'ambito più propriamente scientifico, rivendicando la legittimità e il contributo di scienziati cattolici, nel 1898 la presenza di eventi cattolici nel programma dell'esposizione fece sì che l'attenzione fosse calamitata, oltre che sulla Sindone, di cui si è sopra detto, sulla mostra di arte sacra e su quella delle missioni, in cui i convertiti provenienti da ogni parte del mondo incarnavano lo sforzo della Chiesa e il successo dell'opera evangelizzatrice ${ }^{40}$. Il tema delle missioni apostoliche aveva una valenza ben più ampia rispetto alla conversione degli indigeni, ma coinvolgeva l'intera società italiana e la sua identità politica e culturale. Infatti, come sostiene Guido Abbattista,

la presenza della religione, del sacro e dell'impegno religioso assumeva il significato di una specie di reconquista: riconquista di un terreno fin lì spiritualmente arido e riconquista di capacità di contribuire alla costruzione di una modernità sacralizzata e ri-evangelizzata. Il tema missionario non poteva non ritrovare perciò sua naturale collocazione nel contesto espositivo, in quanto riguardante l'essenza più vera $\mathrm{e}$ profonda della Chiesa, che si esplicava tanto verso il mondo pagano dei popoli selvaggi o comunque ignoranti la parola di Cristo, quanto verso la società europea contemporanea, ancora tanto bisognosa dell'annuncio e dell'esempio cristiano4'

Completavano l'esposizione cattolica il terzo Congresso Mariano, tenutosi nel capoluogo piemontese dal 4 all' 8 settembre, il cui svolgimento fu dettagliatamente riferito dalla «La Civiltà Cattolica»42, e l'inaugurazione del monumento a Don Bosco il 18 settembre alla presenza sia dell'arcivescovo Richelmy, sia di Tommaso Villa43.

Nonostante la polemica iniziale in occasione dell'inaugurazione dell'esposizione laica, «La Civiltà Cattolica» si dedicò a raccontare ed elogiare le manifestazioni

\footnotetext{
38 «La Civiltà Cattolica», anno XLIX, serie XVII, vol. II, 1898, pp. 740-742.

39 Ibidem.

${ }^{40}$ Sul tema delle esposizioni etniche relative non solo ai casi torinesi: Cfr. ABBATTISTA, Guido, Umanità in mostra, cit..

${ }^{41}$ ABBATTISTA, Guido, Umanità in mostra, cit., p. 210.

42 «La Civiltà Cattolica», anno XLIX, serie XVII, vol. IV, 1898, pp. 103-105.

43 «La Civiltà Cattolica», anno XLIX, serie XVII, vol. IV, 1898, pp. 231-232.
} 
cattoliche. Nel 1898, infatti, la carica polemica contro l'esposizione laica e liberale e i suoi animatori risultò (almeno in parte e soprattutto in confronto al 1884) smussata dalla presenza di un programma cattolico, accostato ma non subordinato alle celebrazioni. Proprio la presenza delle iniziative cattoliche fece sbilanciare «La Civiltà Cattolica» fino addirittura a «riconoscere la grandiosità della mostra torinese, e addirittura a esaltarne la natura di "vera città incantata", degna celebrazione del lavoro umano, ammesso che - ci si affrettava a precisare - scopo ultimo del lavoro umano fosse esclusivamente quello di rendere la vita più comoda e agiata»44.

Il successo del 1898 dimostrò la possibilità di superare confini e schemi culturali preesistenti: le due distinte esposizioni, ricomprese nell'Esposizione Generale Italiana, furono sì un caso isolato e unico, ma anche una tentativo, per quanto difficile e non pienamente riuscito, di conciliazione e un'occasione di verifica per i successivi, imminenti equilibri amministrativi e politici, di fronte al dilagare della questione sociale 45.

\section{Conclusioni}

Le esposizioni torinesi qui analizzate rappresentano due casi particolarmente interessanti per delineare l'atteggiamento della «Civiltà Cattolica», e il suo evolversi, sia nei confronti di tematiche scientifiche in senso lato, sia verso un evento divenuto rapidamente un simbolo del positivismo e di una scienza tendenzialmente laica. La rivista dei gesuiti, una delle più autorevoli tribune del pensiero cattolico intransigente $\mathrm{e}$ allineato alla Santa Sede, colse in entrambi i casi l'occasione per rinfocolare la polemica contro il positivismo e la classe dirigente liberale. I messaggi identitari dei quali le esposizioni si facevano portatrici, attraverso l'istituzione di un connubio tra Risorgimento e progresso tecnico-scientifico, furono contrastati: da una parte, nel 1884 i gesuiti presero le distanze dai contenuti politici, volgendosi soprattutto a quelli scientifici, solo in apparenza politicamente neutri, ma utili invece a rivendicare la legittimità e il contributo determinante di ecclesiastici nelle scienze; dall'altra, nel 1898 il convulso contesto politico e sociale e la giustapposizione di un'esposizione cattolica accanto a quella liberale fornirono al periodico ulteriori argomenti per ritrarre e proporre un'identità cattolica, alternativa e preferibile a quella laica e nazionale: l'inserimento di importanti eventi religiosi (come l'ostensione della Sindone e le mostre

\footnotetext{
44 ABBATTISTA, Guido, Umanità in mostra, cit., p. 217.

45 LEVRA, Umberto, ROCCIA, Rosanna (a cura di), Le esposizioni torinesi 1805-1911, cit., p. XIX.
} 
di arte e delle missioni) all'interno del programma di una manifestazione tradizionalmente laica e positivista ne rappresentò infatti una sorta di legittimazione pubblica.

Questa attività di polemica e propaganda non fu però confinata soltanto nel campo politico, ma la scienza, protagonista indiscussa delle esposizioni, svolse sotto questo punto di vista un ruolo rilevante. Il periodo in cui «La Civiltà Cattolica» si occupò dell'esposizioni furono gli anni in cui infuriava la battaglia antievoluzionista e la confutazione di fenomeni (quali ipnotismo, spiritismo, magnetismo) ancora poco chiari, ma che esercitavano un fascino enorme su scienziati positivisti, come Cesare Lombroso e Enrico Morselli. L’intervento della rivista, dunque, non può essere letto riduttivamente nella sola ottica politica. L'attenzione dedicata alle esposizioni intersecava un interesse apologetico e polemico, su cui si basava fin dalla fondazione «La Civiltà Cattolica», nell'ottica di uno sforzo coerente mirante a non escludere la scienza, ma a ricomprenderla, una scienza in armonia (ma, in realtà, sottoposta) alla Rivelazione. Inoltre, non è da sottovalutare il ruolo della scienza nella creazione di un'identità cattolica, così come proprio nella seconda metà dell’Ottocento era accaduto, pur con forza ovviamente maggiore, per liberali e laici. In tutte le questioni scientifiche, dal progresso tecnico al darwinismo, fino alla biopolitica, settori del mondo cattolico, come la rivista dei gesuiti, intervennero non solo per confutare e controbattere agli avversare, ma anche per costruire una scienza cattolica, non retrograda e fatta di erudizione, ma aggiornata e moderna, purché in accordo ai principi cristiani, a cui l'opinione pubblica italiana potesse guardare.

L'idea e l'atteggiamento dei cattolici di fronte alle tematiche scientifiche, e dunque anche alle esposizioni, è complesso e sarebbe riduttivo pensare a un'opposizione cattolica alla scienza e al progresso tecnologico intransigente e completa, così come a un'unica posizione monolitica all'interno del cattolicesimo stesso. I casi delle esposizioni torinesi, qui analizzati alla luce dell'interesse a loro rivolto dalla «Civiltà Cattolica», sono utili in questa prospettiva. Nel 1884 l'avversione verso il positivismo e verso la classe dirigente nazionale e liberale si alimentarono a vicenda, intersecando inoltre il topos della presunta opposizione dei cattolici alle scienze e al loro progresso; nel 1898 i primi segnali di un nuovo clima politico e un'inedita esposizione con "doppia anima”, laica e cattolica determinarono un atteggiamento più inclusivo della rivista verso l'evento. In entrambe i casi, e in ogni dimensione, da quella politica alla scientifica, «La Civiltà Cattolica» perseguì la propria strategia di polemica e apologia, di attacco al laicismo, al positivismo e al liberalismo (già stigmatizzati qualche decennio 
prima dal Sillabo) e di costruzione di un'opinione pubblica e di un'identità cattolica, tanto politica quanto culturale. 


\section{* L'autore}

Carlo Bovolo si è laureato nel 2013 in Storia del Risorgimento all'Università di Torino. Attualmente è dottorando presso l'Università del Piemonte Orientale con un progetto di ricerca sui cattolici e la scienza tra Otto e Novecento attraverso le riviste cattoliche. I suoi interessi di ricerca sono il Risorgimento e il suo uso politico e culturale, i processi di nation building, l'approccio dei cattolici alla scienza. È membro della European Society for the History of Science.

URL: < http://www.studistorici.com/progett/autori/\#Bovolo >

\section{Per citare questo articolo:}

BOVOLO, Carlo, «"Mostrare il moderno". Le Esposizioni universali tra fine Ottocento e gli inizi del Novecento», Diacronie. Studi di Storia Contemporanea : Le esposizioni: propaganda e costruzione identitaria, 29/6/2014, URL: < http://www.studistorici.com/2014/6/29/bovolo_numero_18/ >

\section{Diacronie Studi di Storia Contemporanea $\beta$ www.diacronie.it}

Risorsa digitale indipendente a carattere storiografico. Uscita trimestrale. redazione.diacronie@hotmail.it

Comitato di redazione: Jacopo Bassi - Luca Bufarale - Elisa Grandi - Deborah Paci - Fausto Pietrancosta - Matteo Tomasoni - Luca Zuccolo 